\title{
Chain collapse and aggregation in dilute solutions of poly(methyl methacrylate) below the theta temperature
}

\author{
Yasuyuki Maki
}

Dilute solutions of poly(methyl methacrylate) in some polar solvents show very slow phase separation and remain transparent for long time periods below the cloud point. Because of this slow phase separation, both chain collapse (coil-globule transition) and aggregation were studied systematically with light scattering. In this review, recent findings on chain collapse and aggregation in dilute solutions below the $\theta$-temperature are summarized.

Polymer Journal (2014) 46, 641-645; doi:10.1038/pj.2014.39; published online 28 May 2014

Keywords: aggregation; coil-globule transition; light scattering; memory effect; phase separation

A single polymer chain in a solution under thermodynamically poor solvent conditions assumes a compact globular conformation. ${ }^{1}$ Many theoretical studies have focused on the conformational transition from a random coil to a globule (coil-globule transition) below the $\theta$-temperature. ${ }^{2}$ However, direct observation of the coil-globule transition has been achieved in only a few experimental studies because chain aggregation occurs below the cloud point and obscures the observation of the collapse of each polymer chain. The formation of clusters of polymer chains can be detected by techniques such as light scattering, but the chain aggregation process has not been studied in detail because it occurs too rapidly to be followed quantitatively.

The first attempts to observe the coil-globule transition were conducted on dilute solutions of polystyrene in cyclohexane, a classic theta-solvent system that shows phase behavior with an upper critical solution temperature (UCST). ${ }^{3,4}$ To minimize the effects of aggregation, light scattering experiments were carried out with highly diluted polymer solutions $\left(<10^{-7} \mathrm{~g} \mathrm{~cm}^{-3}\right){ }^{3}$ However, the coil-globule transition curves obtained by different groups seem contradictory. ${ }^{4}$ Recently, experiments on the coil-globule transition were performed for aqueous solutions of $\operatorname{poly}(N$-isopropyl acrylamide) (PNIPAM), which show a lower critical solution temperature (LCST)..$^{5-7}$ Wang et al. ${ }^{7}$ used an extremely dilute $\left(7 \times 10^{-7} \mathrm{~g} \mathrm{~cm}^{-3}\right)$ solution of PNIPAM with a molecular weight $\left(M_{\mathrm{w}}\right)$ of $1.3 \times 10^{7}$ and observed the transition from a random coil to a thermodynamically stable globule. The coil-globule transition curve of PNIPAM was not well-represented by a simple theory based on excluded volume interaction, ${ }^{7}$ most likely owing to the specificity of the LCST-type aqueous solution. In several kinetic studies of the chain collapse process for polystyrene in cyclohexane ${ }^{8}$ and for PNIPAM in water, ${ }^{6}$ the formation of chain aggregates was observed after the chain collapse, but the aggregation processes were not explored. In the case of
PNIPAM in water, small aggregates (mesoglobules ${ }^{9}$ ) were formed immediately after the solutions were heated above LCST. The size of the mesoglobules remained constant over periods of several days, and the solutions did not phase-separate macroscopically during the usual experimental timescales. This peculiar behavior, referred to as colloidal stability, was studied intensively, ${ }^{9}$ but the kinetics of mesoglobule formation was not measured. The colloidal stability of the mesoglobules of PNIPAM is relevant to the 'viscoelastic effect,' discussed elsewhere in this review. ${ }^{10}$

More recently, we illustrated that both chain collapse and aggregation can be studied systematically with dilute solutions of poly(methyl methacrylate) (PMMA) by light scattering experiments. ${ }^{1-18}$ Dilute solutions of PMMA in some polar solvents, such as isoamyl acetate, tert-butyl alcohol (TBA) and acetonitrile (AcN), show very slow phase separation and remain transparent for long time periods, ranging from hours to months, below the cloud point. ${ }^{19}$ Because of this slow phase separation, aggregation processes of PMMA chains resulting from phase separation can be quantitatively measured with light scattering. ${ }^{11,15,16,19}$ For example, an increase in scattered light intensity owing to chain aggregation after a quench to below the cloud point was $\sim 300$ times slower for PMMA in AcN than for polystyrene in cyclohexane. ${ }^{11}$ Moreover, chain collapse kinetics was observed without disturbance by chain aggregation for the PMMA solutions under appropriate conditions. ${ }^{11-15,17-19}$ In this review, recent findings on chain collapse and aggregation in dilute solutions of PMMA below the $\theta$-temperature are summarized.

\section{COIL-GLOBULE TRANSITION OF PMMA}

In studies of the chain collapse process, ${ }^{11-15,17-19}$ dilute solutions of PMMA with high molecular weights $\left(M_{\mathrm{w}} \sim 10^{7}, M_{\mathrm{w}} / M_{\mathrm{n}}=1.2-1.3\right)$ at polymer concentrations $\sim 10^{-4} \mathrm{~g} \mathrm{~cm}^{-3}$ were used for light scattering experiments. The dimensions of polymer chains in the collapse 
process were represented by the expansion factor $\alpha^{2}=\left\langle S^{2}>\right.$ / $\left\langle S^{2}\right\rangle_{0}$, where $\left\langle S^{2}\right\rangle$ and $\left\langle S^{2}\right\rangle_{0}$ are mean-square radius of gyration and $\left\langle S^{2}\right\rangle$ at the $\theta$-temperature, respectively. When the solutions were quenched below the $\theta$-temperature, $\alpha^{2}$ decreased with time and reached an equilibrium value before the determination of $\left\langle S^{2}\right\rangle$ of a single chain was disturbed by the effect of chain aggregation. ${ }^{19}$ The chain collapse processes in PMMA solutions were much slower than those in other polymer systems. ${ }^{6,8,20} \mathrm{~A}$ period of hours to weeks was required for $\alpha^{2}$ to reach its equilibrium value, depending on the solvent species and molecular weight. ${ }^{19}$ Chain collapse became significantly slower for longer PMMA chains. The molecular weight dependence on the rate of chain collapse was investigated for solutions of PMMA with $M_{\mathrm{W}}=6.4 \times 10^{6}$ and $1.1 \times 10^{7}$ in pure $\mathrm{AcN}$ and $\mathrm{AcN}+$ water $(10$ vol\%), respectively. ${ }^{14}$ The characteristic time $\left(\tau_{c}\right)$ of chain collapse was conjectured as $\tau_{\mathrm{c}} \sim M^{z}$, where $M$ is molecular weight. The observed exponent values were $z \sim 3.0$ in pure AcN and $z \sim 2.4$ in $\mathrm{AcN}+$ water. These values were compatible with the theoretical prediction $(z=3)$ based on a phenomenological model. ${ }^{14}$

Plots of the equilibrium values of $\alpha^{2}$ after the collapse process against temperature $T$ revealed the coil-globule transition of PMMA. ${ }^{11,14,19}$ Figure 1 shows the coil-globule transition curves for PMMA in AcN, ${ }^{11} \mathrm{AcN}+$ water $(10 \mathrm{vol} \%),{ }^{14}$ and $\mathrm{TBA}+$ water $(2.5$ vol\%). ${ }^{19,21}$ In Figure 1, the transition curves are represented by plots of $\alpha^{2}$ against a reduced temperature, $(1-\theta / T) M^{1 / 2}$, which are independent of molecular weight but dependent on the nature of the polymer-solvent system. The coil-globule transition was sharp in TBA + water ( 2.5 vol\%) but broad in $\mathrm{AcN}$. The transition curves were well-represented by the theories based on the excluded volume interaction developed by Birshtein and Pryamitsyn ${ }^{22}$ and Grosberg and Kuznetsov. ${ }^{23}$ In the latter theory, chain behavior in the globule state was analyzed based on the Lifshitz model, ${ }^{24}$ and the expansion factor was derived in the form $\alpha=P^{1 / 2} \alpha^{\star}(x)$, where $\alpha^{\star}$ is a reduced function of the reduced temperature $x=Q(1-\theta / T) M^{1 / 2}$, and $P$ and $Q$ are parameters related to the binary and ternary cluster integral among the polymer segments. ${ }^{23}$ The reduced function $\alpha^{*}$ was calculated numerically in the globule region, ${ }^{23}$ and the result was approximated by ${ }^{11}$

$$
P^{3 / 2} \alpha^{-3}=-0.70-0.084 Q(1-\theta / T) M^{1 / 2} .
$$

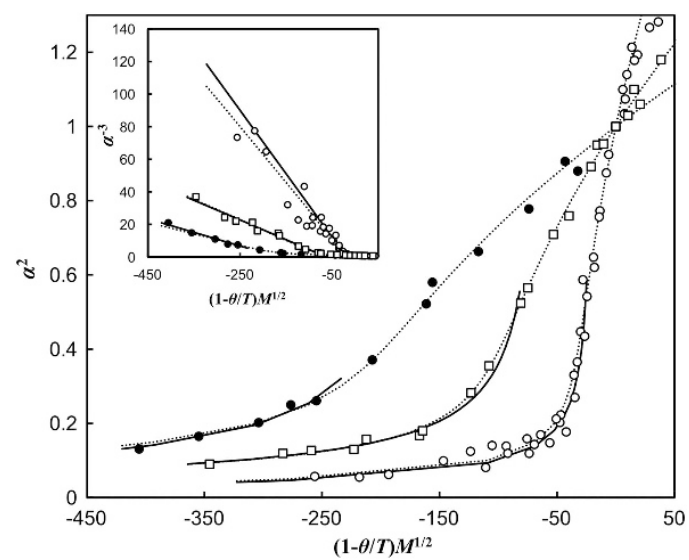

Figure 1 Coil-globule transition curves for PMMA in AcN (filled circles), $\mathrm{AcN}+$ water (10 vol\%) (open squares) and TBA + water (2.5 vol\%) (open circles). Solid and dotted lines were calculated based on the theories of Grosberg and Kuznetsov ${ }^{23}$ and Birshtein and Pryamitsyn, ${ }^{22}$ respectively. The inset shows the same data as the plots of $\alpha^{-3}$ versus $(1-\theta / T) M^{1 / 2}$.
The data for the coil-globule transition curve were plotted in the form of $\alpha^{-3}$ versus (1- $\left.-1 / T\right) M^{1 / 2}$ (inset of Figure 1), and a straight line was fitted to the data points in the globule region, from which the values of $P$ and $Q$ were estimated. The binary and ternary cluster integrals among the polymer segments were calculated from the values of $P$ and $Q$, and the obtained values were consistent with the literature values ${ }^{25}$ derived from the second and third virial coefficients of the dilute solution. ${ }^{11}$

\section{MEMORY EFFECT IN THE CHAIN COLLAPSE PROCESS}

Many theoretical studies on the chain collapse process after a quench were carried out based on phenomenological models. Grosberg et al. ${ }^{26}$ predicted a two-stage collapse process in which fast crumpling of an unknotted polymer chain occurs and slow knotting via reptation-like chain motion in the crumpled globule follows to form a more compact globule. Several other groups ${ }^{27}$ considered a different process in which a 'pearl-necklace' structure consisting of globular droplets of a dense phase connected by strings is formed quickly and coalescence of the droplets subsequently occurs. In examining the equilibrium and non-equilibrium structure of a globule, one effective approach is to observe the responses to temperature perturbation applied to the globule. ${ }^{28,29}$ To verify knot formation in a globule, ${ }^{26}$ chain expansion from globule to coil were measured for PMMA in TBA + water $(2.5$ vol\%). ${ }^{12,17,18}$ In these experiments, the PMMA solutions were aged at a temperature below the $\theta$-temperature for a certain period of time and were then rapidly heated to the $\theta$-temperature. The dependence of aging time and molecular weights on the chain expansion rate indicated that the disentanglement process of knotted globules dominated the chain expansion process. ${ }^{12,17}$

Furthermore, the effect of smaller temperature perturbation on globules in the collapse process was investigated by a three-stage temperature shift experiment. ${ }^{13}$ In the temperature shift experiment (inset of Figure 2a), the temperature of dilute solutions of PMMA $\left(M_{\mathrm{w}}=1.1 \times 10^{7}\right)$ in TBA + water $(2.5 \mathrm{vol} \%)$ was changed from the $\theta$-temperature $\left(41.5^{\circ} \mathrm{C}\right)$ to a temperature $T_{1}$ at time $t=0$, then from $T_{1}$ to $T_{2}$ at $t=t_{1}$, and finally from $T_{2}$ to $T_{1}$ at $t=t_{1}+t_{2}$. Figure $2 \mathrm{a}$ shows plots of $\alpha^{2}$ versus $t$ in the chain collapse processes for temperature shift experiments with a negative temperature perturbation $\left(T_{1}>T_{2}\right)$. In the second stage, gradual relaxation in $\alpha^{2}$ was observed after a sudden decrease induced by the temperature drop. In the third stage, $\alpha^{2}$ changed in two steps, a temporal increase caused by the temperature increase and a subsequent slow decrease to its equilibrium value. Interestingly, as shown in Figure $2 b$, the chain collapse processes observed in the first and third stages were connected smoothly and agreed with the collapse process owing to a single-stage quench to $T_{1}$ applied at $t=0$. This behavior was reminiscent of a memory effect in the aging of glassy materials below the glass transition temperature. ${ }^{30-32}$ It is not obvious if chain motion in the globule is frozen like glass because the segment volume fraction in the globule is less than $0.2 .{ }^{13}$ From a phenomenological viewpoint, it is conjectured that if there exist at least two processes responding to the temperature shift and characteristic times of the processes that are totally different from each other, the memory effect appears because the faster process represents the temporal response to the temperature change and the slower process serves as 'memory' of the whole process. In Figure $2 \mathrm{~b}$, the deviation of the data for $T_{2}=27^{\circ} \mathrm{C}$ from the curve for the collapse process at $T_{1}\left(=30^{\circ} \mathrm{C}\right)$ owing to a singlestage quench was larger than that for $T_{2}=22^{\circ} \mathrm{C}$. From the above phenomenological viewpoint, this behavior would be attributed to acceleration of the slower process relevant to 'memory' for the higher 
a

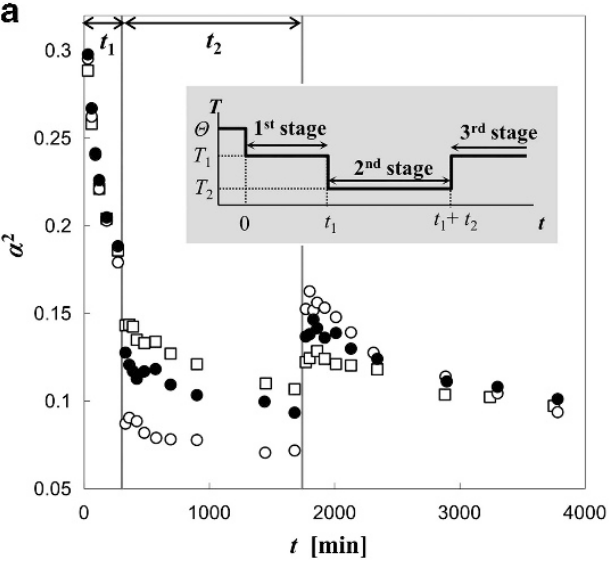

b

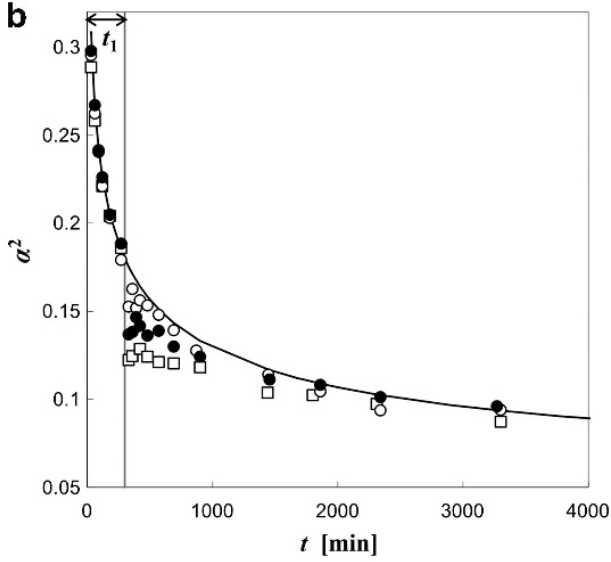

Figure 2 (a) Plots of $\alpha^{2}$ versus $t$ for the chain collapse process subjected to negative temperature perturbation at $t_{1}=300$ min and $t_{1}+t_{2}=1740$ min. The temperature $T_{1}$ in the first and third stages is $30^{\circ} \mathrm{C}$, and the temperatures $T_{2}$ in the second stage are $22^{\circ} \mathrm{C}$ (open circles), $25^{\circ} \mathrm{C}$ (filled circles) and $27^{\circ} \mathrm{C}$ (open squares). The inset shows a schematic illustration of the three-stage temperature shift experiment. (b) Plots obtained by omitting the plots in the second stage and connecting the plots in the first and third stages in a. A solid line represents the collapse process owing to the single quench to $30^{\circ} \mathrm{C}$ applied at $t=0$.
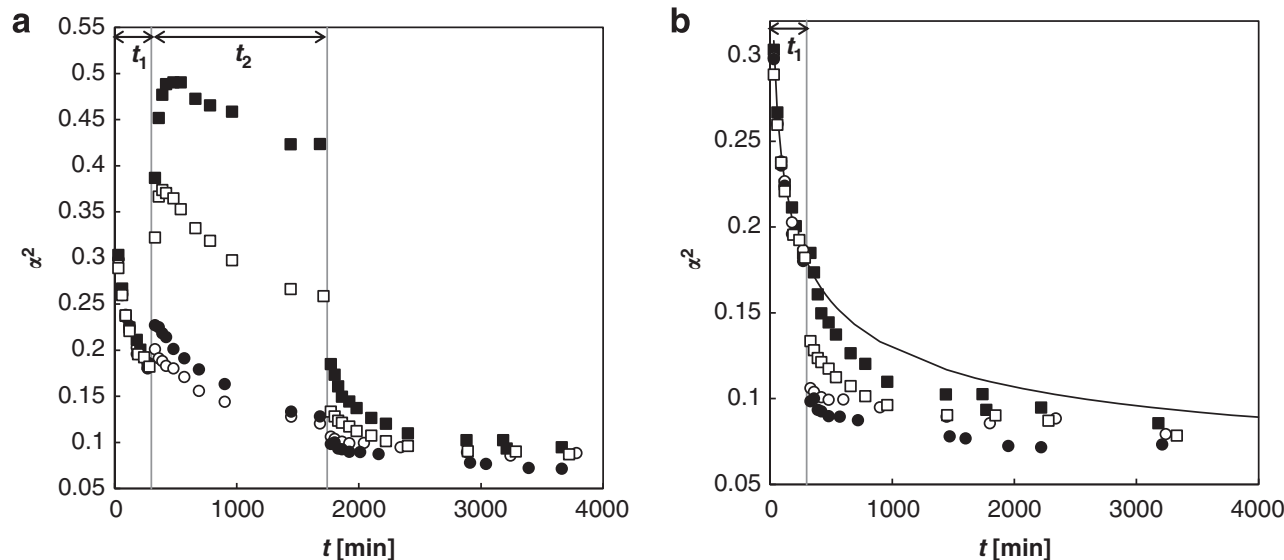

Figure 3 (a) Plots of $\alpha^{2}$ versus $t$ for the chain collapse process subjected to positive temperature perturbation at $t_{1}=300 \mathrm{~min}$ and $t_{1}+t_{2}=1740 \mathrm{~min}$. The temperature $T_{1}$ in the first and third stages is $30^{\circ} \mathrm{C}$, and the temperatures $T_{2}$ in the second stage are $32{ }^{\circ} \mathrm{C}$ (open circles), $34^{\circ} \mathrm{C}$ (filled circles), $37^{\circ} \mathrm{C}$ (open squares) and $38^{\circ} \mathrm{C}$ (filled squares). (b) Plots obtained by omitting the plots in the second stage and connecting the plots in the first and third stages in a. A solid line represents the collapse process owing to the single quench to $30^{\circ} \mathrm{C}$ applied at $t=0$.

$T_{2}$. Investigations on molecular processes involved in the memory effect in the chain collapse process are now in progress.

Figures $3 \mathrm{a}$ and $4 \mathrm{a}$ illustrate chain collapse processes in temperature shift experiments with a positive temperature perturbation $\left(T_{1}<T_{2}\right)$. For smaller temperature perturbations (Figure $3 a$ ), in the second stage, $\alpha^{2}$ increased instantaneously in response to the temperature rise, and chain contraction occurred after the overshooting chain expansion. In the third stage, $\alpha^{2}$ suddenly decreased owing to the temperature drop, followed by slow relaxation. For larger temperature perturbations (Figure 4a), $\alpha^{2}$ increased immediately after the temperature rise and soon reached its equilibrium value in the second stage. In the third stage, a chain collapse process occurred in a similar manner as in the first stage. Figures $3 \mathrm{~b}$ and $4 \mathrm{~b}$ show plots obtained by omitting the plots in the second stage and connecting the plots in the first and third stages of Figures $3 \mathrm{a}$ and $4 \mathrm{a}$, respectively. Figure $3 \mathrm{~b}$ shows that an acceleration of the chain collapse was caused by smaller positive temperature perturbations, and that in a sense, 'over-aging' 31 of the globule was induced by the temperature perturbation. However, Figure $4 \mathrm{~b}$ indicates that the chain collapse process was decelerated by larger positive temperature perturbations; 'rejuvenation $^{33}$ of the globule apparently occurred. In these large positive temperature perturbations, the temperatures in the second stage are higher than the coil-globule transition temperature $\left(\sim 39^{\circ} \mathrm{C}\right.$ for PMMA $\left(M_{\mathrm{w}}=1.1 \times 10^{7}\right)$ in TBA + water $\left.(2.5 \mathrm{vol} \%)\right)^{21}$. Because the temperature history of the globular chain was lost when the chain expanded to assume a random coil conformation in the second stage, the collapse processes in the third stage in Figure $4 \mathrm{~b}$ agreed with the collapse process owing to the single quench applied at $t=t_{1}$ (dotted line).

\section{COMPETITION BETWEEN CHAIN COLLAPSE AND AGGREGATION}

When a dilute polymer solution is quenched below the cloud point, chain collapse and aggregation occur competitively because these phenomena are driven by the same interaction between polymer segments both intra and intermolecularly. ${ }^{34,35}$ This view has been clarified by recent studies on PMMA solutions below the cloud point. ${ }^{14-16}$ 

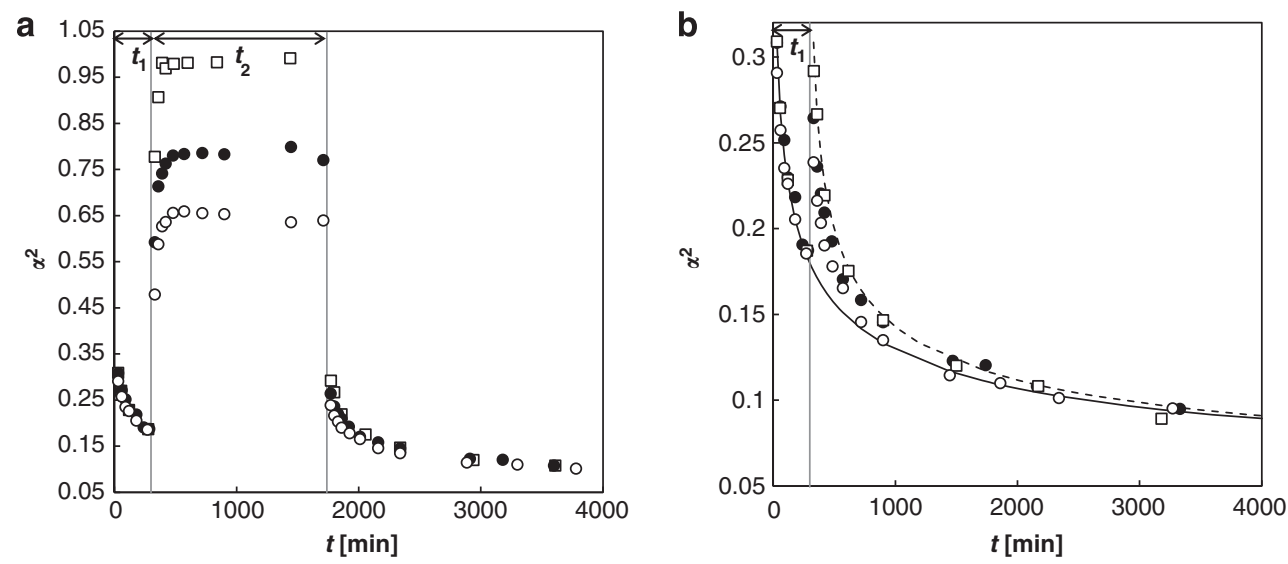

Figure 4 (a) Plots of $\alpha^{2}$ versus $t$ for the chain collapse process subjected to positive temperature perturbation at $t_{1}=300 \mathrm{~min}$ and $t_{1}+t_{2}=1740 \mathrm{~min}$. The temperature $T_{1}$ in the first and third stages is $30^{\circ} \mathrm{C}$, and the temperatures $T_{2}$ in the second stage are $39^{\circ} \mathrm{C}$ (open circles), $40^{\circ} \mathrm{C}$ (filled circles) and $41.5^{\circ} \mathrm{C}$ (open squares). (b) Plots obtained by omitting the plots in the second stage and connecting the plots in the first and third stages in a. Solid and dotted lines represent the collapse processes owing to the single quench to $30^{\circ} \mathrm{C}$ applied at $t=0$ and at $t=300 \mathrm{~min}\left(=t_{1}\right)$, respectively.

Solvent effects on the kinetics of chain aggregation were compared with that of chain collapse for PMMA in $\mathrm{AcN}$ and in the mixed solvent of $\mathrm{AcN}+$ water (10 vol\%). ${ }^{14,16}$ Chain collapse in $\mathrm{AcN}+$ water was found to occur much faster than that in pure $\mathrm{AcN}$, although the former collapse process required several hours. ${ }^{14}$ The ratio of the characteristic collapse rate in $\mathrm{AcN}+$ water to that in pure AcN was estimated to be $\sim 11 .{ }^{14}$ The chain aggregation processes were measured for the same systems with comparable quench depth and concentrations. The ratio of the aggregation rate in $\mathrm{AcN}+$ water to that in pure AcN was estimated to be $\sim 9 .^{16}$ These similar ratios indicated the correlation between the rates of chain collapse and aggregation and that the nature of the solvent affected the rates of these processes through a similar mechanism.

Molecular weight is also an important factor for the kinetics of chain collapse and aggregation. ${ }^{14,15}$ Earlier studies of the chain aggregation of PMMA in dilute solutions reported that the weightaverage molecular weight $M_{\mathrm{w} \text {,agg }}$ of PMMA clusters in the aggregation process increased exponentially with time, that is, $\ln M_{\mathrm{w}, \mathrm{agg}} \sim g c t$, where $g$ and $c$ are the specific rate of chain aggregation and polymer concentration, respectively. ${ }^{19}$ The molecular weight dependence on aggregation rate $g$ was estimated to be $g \sim M^{-3.0}$ for PMMA in TBA + water $(2.5 \mathrm{vol} \%) .{ }^{15}$ This result suggested that the characteristic times of chain collapse and aggregation have similar molecular weight dependences $\sim M^{3}{ }^{14,15}$

Because the effects of solvent species and molecular weight on collapse kinetics are correlated with those on aggregation kinetics, the competition between chain collapse and aggregation is dominated by polymer concentration. The rate of chain aggregation decreases with concentration, while the rate of chain collapse does not depend on the concentration. At low concentrations, polymer chains would collapse first, and the collapsed chains would aggregate, forming clusters of polymer chains. In previous studies of dilute solutions of PMMA, the collapse process was measured before the onset of chain aggregation for PMMA with higher molecular weights. ${ }^{11,13,14,18,19}$ However, the collapse process was not observed, and the aggregation process of the collapsed chains was measured for PMMA with lower molecular weights ${ }^{16,19}$ on account of the faster collapse for shorter chains. Recently, the successive occurrence of chain collapse and aggregation was measured by an experiment carried out over a long time period (19 weeks). ${ }^{15}$ The entire process of shifting from chain collapse to aggregation was represented by the superposition of each process, although the overlapping of the processes became noticeable at higher concentrations.

The strong molecular weight dependence of the aggregation rate, $g \sim M^{-3}$, can be interpreted in terms of the rheological properties of PMMA clusters formed in the aggregation process. ${ }^{15}$ Cluster formation owing to phase separation occurs much more slowly than the prediction based on the characteristic time of the diffusion-limited cluster aggregation (DLCA) process. $^{8}$ The exponential growth of clusters observed in the aggregation process of PMMA chains is characteristic of the reaction-limited cluster aggregation (RLCA) process. ${ }^{36}$ In DLCA, each collision of two clusters gives rise to a coalescence of the two, but in RLCA, only a small fraction of collisions yields coalescence of the clusters. According to the viscoelastic effect proposed by Tanaka ${ }^{37}$ to explain the slow phase separation in dilute polymer solutions, two colliding clusters coalesce when polymer chains can interdiffuse between two clusters during a contact. This concept was represented by two characteristic times: a chain diffusion time $\tau_{\text {rep }}$ and a contact time $\tau_{\text {cont }}$. For $\tau_{\text {rep }} / \tau_{\text {con }} \gg 1$, chains rarely bridge the two clusters during a contact, and aggregation occurs via RLCA mechanism. For $\tau_{\text {rep }} / \tau_{\text {cont }} \ll<1$, the coalescence of clusters occurs at each collision, and aggregation occurs via DLCA mechanism. The two characteristic times were estimated as $\tau_{\text {rep }} \sim a^{2} N^{3} \varphi^{3 / 2} / D_{1}$ for the reptation of a chain in a dense polymer matrix and as $\tau_{\text {cont }} \sim r_{0}^{2} / D_{\mathrm{R}}$ for to the Brownian motion of a cluster, where $a$ is the length of monomer, $N$ is the degree of polymerization, $\varphi$ is the volume fraction of polymer, $r_{0}$ is the range of interaction, and $D_{1}$ and $D_{\mathrm{R}}$ are the diffusion constants of a monomer and a cluster, respectively. ${ }^{37}$ In the aggregation process of PMMA in TBA + water (2.5 vol $\%)$, the value of $\tau_{\text {rep }} / \tau_{\text {cont }}$ was estimated as $\tau_{\text {rep }} / \tau_{\text {cont }}>10^{6}$; thus, the condition for reaction-limited cluster aggregation $\left(\tau_{\text {rep }} /\right.$ $\tau_{\text {cont }} \gg 1$ ) was satisfied. ${ }^{15}$ If the aggregation process is dominated by $\tau_{\text {rep }}\left(\sim N^{3}\right)$ in the case of $\tau_{\text {rep }} / \tau_{\text {cont }} \gg 1$, the aggregation rate is predicted to be $g \sim N^{-3}$, which is consistent with the observed result. The rheological aspects in chain aggregation were also observed in the temperature dependence of the rate of the chain aggregation of $\mathrm{PMMA}^{19}$ and are a subject of future research.

\section{CONCLUSIONS}

Both the coil-globule transition and chain aggregation can be quantitatively studied for dilute solutions of PMMA by light scattering experiments because of the slow phase separation of the PMMA 
solutions. The coil-globule transition was measured for PMMA in various solvents, and the obtained coil-globule transition curve was well-represented by theories based on excluded volume interactions. The structure of the globule was examined by applying temperature perturbations to the PMMA solutions below the $\theta$-temperature. A phenomenon analogous to the memory effect of glassy materials was observed in the chain collapse process subjected to negative temperature perturbations. A process shifting from chain collapse to chain aggregation was measured for PMMA solutions below the cloud point, which clarified the competitive occurrence of the collapse and aggregation processes. The strong molecular weight dependence on the rate of chain aggregation owing to phase separation was interpreted in terms of the viscoelastic properties of clusters of PMMA chains formed in the aggregation process.

\section{CONFLICT OF INTEREST}

The authors declare no conflict of interest.

\section{ACKNOWLEDGEMENTS}

This work was partially supported by KAKENHI (Grant-in-Aid for Scientific Research) on the priority Area 'Soft Matter Physics' from the Ministry of Education, Culture, Sports, Science and Technology of Japan.

1 Grosberg, A. Yu. \& Khokhlov, A. R. Statistical Physics of Macromolecules (AIP Press, New York, 1994).

2 Baysal, B. M. \& Karasz, F. E. Coil-globule collapse in flexible macromolecules. Macromol. Theory Simul. 12, 627-646 (2003).

3 Sun, S.-T., Nishio, I., Swislow, G. \& Tanaka, T. The coil-globule transition: Radius of gyration of polystyrene in cyclohexane. J. Chem. Phys. 73, 5971-5975 (1980).

4 Park, I. H., Wang, Q.-W. \& Chu, B. Transition of linear polymer dimensions from $\theta$ to collapsed regime. 1. Polystyrene/cyclohexane system. Macromolecules 20, 1965-1975 (1987).

5 Kubota, K., Fujishige, S. \& Ando, I. Single-chain transition of poly( $\mathrm{N}$-isopropylacrylamide) in water. J. Phys. Chem. 94, 5154-5158 (1990).

6 Wu, C. \& Zhou, S. Laser light scattering of the phase transition of $\operatorname{poly}(N$ isopropylacrylamide) in water. 1. Single chain. Macromolecules 28, 8381-8387 (1995).

7 Wang, X., Qiu, X. \& Wu, C. Comparison of the coil-to-globule and globule-to-coil transition of a single poly( $\mathrm{N}$-isopropylacrylamide) homopolymer chain in water. Macromolecules 31, 2972-2976 (1998).

8 Chu, B., Ying, Q. \& Grosberg, A. Yu. Two-stage kinetics of single-chain collapse. Polystyrene in cyclohexane. Macromolecules 28, 180-189 (1995).

9 Aseyev, V. O., Tenhu, H. \& Winnik, F. M. Temperature dependence of the colloidal stability of neutral amphiphilic polymers in water. Adv. Polym. Sci. 196, 1-85 (2006).

10 Zhang, G. \& Wu, C. Folding and formation of mesoglobules in dilute copolymer solutions. Adv. Polym. Sci. 195, 101-176 (2006).

11 Maki, Y., Sasaki, N. \& Nakata, M. Coil-globule transition of poly(methyl mechacrylate) in acetonitrile. Macromolecules 37, 5703-5709 (2004).

12 Nakata, M., Nakamura, Y., Maki, Y. \& Sasaki, N. Slow expansion of a single polymer chain from the knotted globule. Macromolecules 37, 4917-4921 (2004).
13 Maki, Y., Sasaki, N. \& Nakata, M. Memory effect in the chain-collapse process in a dilute polymer solution. J. Chem. Phys. 121, 12690-12695 (2004).

14 Maki, Y., Dobashi, T. \& Nakata, M. Kinetics of chain collapse in dilute polymer solutions: molecular weight and solvent dependences. J. Chem. Phys. 126, 134901 (2007).

15 Nakata, M., Nakamura, Y., Sasaki, N. \& Maki, Y. Transition from a chain-collapse process to a chain-aggregation process of poly(methyl methacrylate) in a mixed solvent. Phys. Rev. E 76, 041805 (2007)

16 Maki, Y., Dobashi, T. \& Nakata, M. Comparison of the kinetics of chain aggregation and chain collapse in dilute polymer solutions. Phys. Rev. E 78, 041802 (2008).

17 Nakata, M., Nakamura, Y., Sasaki, N. \& Maki, Y. Slow knot formation by suppressed self-reptation in a collapsed polymer chain. Phys. Rev. E 85, 021802 (2012).

18 Maki, Y. \& Dobashi, T. Light scattering of dilute solutions of poly(methyl methacrylate) below the theta temperature. Kobunshi Ronbunshu 69, 373-381 (2012).

19 Maki, Y. in Nano/Micro Science and Technology in Biorheology: Principles, Methods, and Applications (eds Dobashi, T. \& Kita, R.) Ch. 2 (in press).

20 Ye, X., Lu, Y., Shen, L., Ding, Y., Liu, S., Zhang, G. \& Wu, C. How many stages in the coil-globule transition of linear homopolymer chains in a dilute solution? Macromolecules 40, 4750-4752 (2007)

21 Nakamura, Y., Sasaki, N. \& Nakata, M. Kinetics of the coil-globule transition of poly(methyl methacrylate) in a mixed solvent. Macromolecules 34, 5992-6002 (2001).

22 Birshtein, T. M. \& Pryamitsyn, V. A. Coil-globule type transition in polymers. 2. Theory of coil-globule transition in linear macromolecules. Macromolecules 24, 1554-1560 (1991).

23 Grosberg, A. Yu. \& Kuznetsov, D. V. Quantitative theory of the globule-to-coil transition. 1. Link density distribution in a globule and its radius of gyration. Macromolecules 25 , 1970-1979 (1992).

24 Lifshitz, I. M., Grosberg, A. Yu. \& Khokhlov, A. R. Some problems of statistical physics of polymer chains with volume interaction. Rev. Mod. Phys. 50, 683-713 (1978).

25 Abe, F., Einaga, Y. \& Yamakawa, H. Second virial coefficient of oligo- and poly(methyl methacrylate)s near the $\theta$ temperature. Macromolecules 26, 694-700 (1995).

26 Grosberg, A. Yu., Nechaev, S. K. \& Shakhnovich, E. I. The role of topological constraints in the kinetics of collapse of macromolecules. J. Phys. 49, 2095-2100 (1988).

27 Halperin, A. \& Goldbart, P. M. Early stages of homopolymer collapse. Phys. Rev. E 61, 565-573 (2000).

28 Rabin, Y., Grosberg, A. Yu. \& Tanaka, T. Metastable globules in good solvents: topologically stabilized state of polymers. Europhys. Lett. 32, 505-510 (1995).

29 Lee, N.-K., Abrams, C. F., Johner, A. \& Obukhov, S. Swelling dynamics of collapsed polymers. Macromolecules 37, 651-661 (2004).

30 Fukao, K. \& Sakamoto, A. Aging phenomena in poly(methyl methacrylate) thin films: memory and rejuvenation effects. Phys. Rev. E 71, 041803 (2005).

31 Bellon, L., Ciliberto, S. \& Laroche, C. Temperature cycling during the aging of a polymer glass. Preprint at http://arXiv.cond-mat/9905160.

32 Lefloch, F., Hammann, J., Ocio, M. \& Vincent, E. Can aging phenomena discriminate between the droplet model and a hierarchical description in spin glasses? Europhys. Lett. 18, 647-652 (1992).

33 Vincent, E., Bouchaud, J. P., Hammann, J. \& Lefloch, F. Contrasting effects of field and temperature variations on ageing in spin glasses. Phil. Mag. B 71, 489-500 (1995).

34 Grosberg, A. Yu. \& Kuznetsov, D. V. Single-chain collapse or precipitation? Kinetic diagram of the states of a polymer solution. Macromolecules 26, 4249-4251 (1993).

35 Raos, G. \& Allegra, G. Chain collapse and phase separation in poor-solvent polymer solutions: A unified molecular description. J. Chem. Phys. 104, 1626 (1996).

36 Vicsek, T. Fractal Growth Phenomena (World Scientific, Singapore, 1989).

37 Tanaka, H. Appearance of a moving droplet phase and unusual networklike or spongelike patterns in a phase-separating polymer solution with a double-well-shaped phase diagram. Macromolecules 25, 6377-6380 (1992).

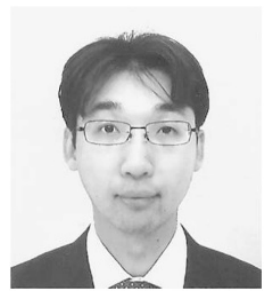

Dr Yasuyuki Maki was born in Hokkaido, Japan in 1978. He received his Doctoral degree of Science in 2005 from the Hokkaido University. He has been an assistant professor at the Gunma University since 2005. His research interests include conformation, association behaviors and gelation of synthetic and biological macromolecules in solutions by use of scattering methods. He was a recipient of Young Scientist Award at the 35th Annual Meeting of Japanese Society of Biorheology in 2012 (the Japanese Society of Biorheology) and Award for Encouragement of Research in Polymer Science in 2012 (The Society of Polymer Science, Japan). 